\title{
The transition from quasar radio-loud to radio-quiet state in the framework of the black hole scalability hypothesis
}

\author{
A. Marecki and B. Swoboda
}

\author{
Toruń Centre for Astronomy, N. Copernicus University, 87-100 Toruń, Poland \\ e-mail: amr@astro.uni.torun.pl
}

Received 23 July 2010 / Accepted 23 September 2010

\begin{abstract}
Aims. There are several lines of evidence that active galactic nuclei (AGN) can be regarded as scaled-up X-ray binaries (XRB). The timescales of the evolutionary phenomena in these two classes are proportional to the black hole (BH) masses. Consequently, unlike in the case of XRBs, the evolution of AGNs is too slow to be followed directly. What could be done, however, is to assign particular types of active galaxies to different evolutionary stages observable in XRBs. We studied such an assignment for three quasars with clear signatures of a recent transition from the radio-loud to the radio-quiet state.

Methods. The quasars we investigated have large-scale radio lobes that are clearly asymmetric - one lobe is of Fanaroff-Riley II type, while the other one is a diffuse relic devoid of a hotspot. We suggest that the prime cause of the asymmetry of these radio sources is that the nuclei of their host galaxies currently produce no jets. To prove that, we observed them with milliarcsecond resolution to check if they are similar to those in radio-quiet quasars.

Results. The observations carried out with the EVN revealed that the nuclei of the quasars under investigation are not of a core-jet type that is characteristic for radio-loud, lobe-dominated quasars. It follows that the lobes are no longer fuelled and that the apparent asymmetry results from the orientation, which causes a time lag of the order of $10^{6}$ years between their images: the lobe perceived as a relic is nearer than the lobe with a hotspot and so it is observed in a later stage of the decay.

Conclusions. The three AGNs under investigation were radio-loud earlier, but now they have switched to the radio-quiet state. In the framework of the XRB/AGN unification, the above means that they have left the very high state and have moved now to the high/soft state. If this scenario is correct it poses a challenge to the so-called spin paradigm. While a radio-loud AGN must have a spinning BH in its centre, the BH of a radio-quiet AGN does not necessarily have low spin; AGNs with high-spin BHs, like those we deal with here, may become radio-quiet.
\end{abstract}

Key words. radio continuum: galaxies - galaxies: active - X-rays: binaries

\section{Introduction}

In mathematical terms, black holes (BHs) are very simple objects. In principle, only two parameters, mass and spin, are sufficient to describe them fully. Consequently, various similarities and analogies between different classes of astronomical objects containing a BH in their centres seem to be likely. Masses of BHs encountered in X-ray binaries (XRBs) on the one extreme and active galactic nuclei (AGNs) on the other span over eight orders of magnitude or more, and it is of interest whether any scaling laws connecting objects of otherwise quite different types like XRBs and AGNs exist - see e.g. Fender et al. (2007) for a review. For the first time, a suggestion that this scaling actually takes place was made by Shakura \& Sunyaev (1976). A corollary of a possible unification of objects with accreting BHs based on their scalability is of great importance because it paves the way for the extrapolation of some observable phenomena in one domain of BHs, namely stellar-mass BHs, to the other, i.e. to the domain of supermassive BHs (SMBHs) encountered in AGNs. Given that in particular characteristic timescales should be proportional to the mass, slow processes that are ongoing in AGNs could be directly followed in XRBs.

The first spectacular manifestation of putative $\mathrm{BH}$ scalability was discovered by Mirabel et al. (1992) who observed the X-ray source 1E 1740.7-2942 with the Very Large Array (VLA) and found its radio structure to be a double-sided jet straddling a compact and variable core. A striking similarity between the appearance of this phenomenon and that of radio-loud quasars (RLQs) led to coining the term "microquasar" for jet-producing XRBs (Mirabel et al. 1992). However, this was GRS 1915+105 - a highly variable XRB containing a $\mathrm{BH}$ of $\sim 15 M_{\odot}-$ which became an archetypal microquasar, see the review by Fender \& Belloni (2004). It was discovered on 1992 August 15 in the X-ray domain (Castro-Tirado et al. 1992) and a few months later in radio (Mirabel et al. 1993). The most exciting property of this object was revealed when Mirabel \& Rodríguez (1994) resolved its radio structure down to the fraction of an arcsecond with the VLA and detected several components moving either toward the east or west away from the core of GRS $1915+105$ with apparently superluminal velocities. By the time of the announcement of that ground-breaking discovery, apparent superluminal motion (SLM) was already well known, but this phenomenon was pertinent entirely to AGNs. Thus, finding the SLM in the vicinity of a Galactic BH not only rendered the term "microquasar" most appropriate for GRS $1915+105$ but also provided hard proof for the existence of an XRB vs. AGN analogy.

This analogy was further confirmed by Marscher et al. (2002), who monitored X-ray and radio emission of AGN in $3 \mathrm{C} 120$ radio galaxy and found dips in the X-ray emission that were followed by ejections of bright superluminal knots in the radio jet, just like in microquasars where dips in the X-ray emission precede radio flares. What was more, the mean time 
between the X-ray dips appeared to scale roughly with the $\mathrm{BH}$ mass. An extension of that monitoring programme carried out by the same group over five years (2002-2007) included observations at X-ray (2-10 keV), optical ( $R$ and $V$ bands), and radio (14.5 and $37 \mathrm{GHz}$ ), as well as imaging with the Very Long Baseline Array (VLBA) at $43 \mathrm{GHz}$ (Chatterjee et al. 2009). The results of this comprehensive investigation were compelling enough to formulate a paradigm that XRBs and both radio-loud and radio-quiet active galactic nuclei were fundamentally similar systems, with characteristic time and size scales linearly proportional to the mass of the central $\mathrm{BH}$. Also, the jet generation mechanism in microquasars and quasars seems to be the same, following the work of Türler et al. (2004), who showed that the variability pattern of GRS $1915+105$ can be reproduced by the standard shock model used for extragalactic jets, e.g. that in $3 \mathrm{C} 273$. Hence, the physical nature of relativistic jets does not depend on the mass of the $\mathrm{BH}$ and consequently the study of jets in both quasars and microquasars is complementary. In line with the above results, McHardy et al. (2006) showed that characteristic timescales in the X-ray variations both in AGNs and Galactic BHs could be physically linked to an extent that it appeared that the accretion process was exactly the same for small and large BHs. Therefore, over a range $\sim 10^{8}$ in mass and $\sim 10^{3}$ in accretion rate, SMBHs can be regarded as scaled-up Galactic BHs.

However, as far as the scope of this work is concerned, perhaps the most interesting aspect of the XRB/AGN analogy was pointed to by Nipoti et al. (2005). According to them, the well-known radio-loud (RL) vs. radio-quiet (RQ) dichotomy in quasars was reflected in certain states of radio/X-ray emission observable in microquasars: the fraction of observable RLQs $f_{\mathrm{Q}} \approx 0.08-$ is compatible with the fraction of the time that microquasars remain in the so-called flaring state. Moreover, the authors posited that QSOs could switch between the RL and RQ states, but spend much more time in the latter one. This conjecture is testable observationally and the most straightforward way to show that it is correct is to find objects that are currently in a transition between RL and RQ states or are at least shortly after such a transition. At first sight, this seems hardly possible because the timescales of the evolutionary processes in AGNs are too extended for these phenomena to be directly observed. Yet we show here that these tests are possible and present the results we obtained for three QSOs with signatures of a recent transition from the RL to the RQ state. Based on our results, we link this transition with its XRB analog.

\section{The method}

The well known upper limit for the spectral ages of double radio sources is of the order of $\sim 10^{8} \mathrm{yr}$ (Alexander \& Leahy 1987; Liu et al. 1992) and the upper limit for their linear sizes is $\sim 4 \mathrm{Mpc}$, see e.g. Machalski et al. (2008). These two limits constitute compelling evidence that the energy transport from the RL AGN to its lobes cuts off eventually, and so the radio source fades out. The spectrum of a switched-off source becomes very steep because of radiation and expansion losses, and its lobes gradually disperse. According to Komissarov \& Gubanov (1994), the coasting phase of the lobes of a source that is no longer fuelled by its AGN can be very long: up to $10^{8} \mathrm{yr}$. But hotspots fade out much sooner; their lifetimes are roughly $7 \times 10^{4} \mathrm{yr}$ (Kaiser et al. 2000). Hence, the lack of well defined hotspots is an early signature of a lobes' decay.

There is yet another important observable phenomenon resulting from cessation of the activity in the radio domain: the lobe asymmetry, which, as pointed out by Marecki et al. (2006), is a possible signature of a radio activity switch-off. We mean here by "asymmetry" that one lobe is diffuse and devoid of a hotspot, while the other resembles a Fanaroff-Riley (Fanaroff \& Riley 1974) type-II (FR II) lobe quite well. In the extreme case, not only the hotspot but the whole lobe disappears. We interpret this as follows. If a double structure of a radio source does not lie in the sky plane (but also is not beamed towards us) the light-travel time plays a role: the epoch in which we observe the far-side lobe is significantly earlier than the epoch of the nearside lobe. For example, if the angle between the line of sight and the source axis is $45^{\circ}$, the time lag is equal to the projected linear size divided by $c$ and attains a magnitude of the order of $\sim 10^{6}$ years for a typical large-scale, i.e. $\sim 10^{5}$-pc sized radio source. This is definitely long enough for a hotspot to disappear as well for a lobe to disperse considerably. The above means that the lobe asymmetry is a very valuable signature of a recent cessation of RL state or - to be more exact - either a switch-off of the whole activity in the nucleus of the galaxy or at least of a transition from RL to RQ state. To prove that the latter scenario is correct, one has to show radio sources with asymmetric, i.e. one "still alive" and one "dying" lobe straddling a QSO. This way, one proves that the host galaxy is still active but its nucleus no longer produces jets, and so it has entered an evolutionary track leading to the fully RQ state. The presence of broad emission lines, which are best observed in QSOs, is essential to ascertain that in accordance with the AGN unification scheme (Antonucci 1993; Urry \& Padovani 1995), the AGN axis makes a moderate, i.e. neither small nor close to right, angle with the line of sight so that the time lag between the lobes' images indeed takes place, and this is the most plausible and natural explanation of their apparent asymmetry. Using QSOs for this purpose is even more justified by the seminal work of Barthel (1989), who assert that the axis of a QSO makes an angle of less than $44^{\circ} .4$ with the line of sight. Therefore, if a transition from RL to RQ state occurs in a QSO with a large-scale, double-lobed structure, it must result in this asymmetry. To make the proof complete, one should finally show that the high-resolution radio image of the object's core has the characteristics of those observed in "true" RQ quasars (RQQ) known from the literature. Below we report how we carried out the above-sketched proof.

As a first step, we constructed a genuine sample of AGNs that have just switched off in the radio domain. The procedure was as follows. We selected double radio sources as seen in NRAO/VLA Sky Survey (NVSS) (Condon et al. 1998) ${ }^{1}$ with optical identifications located between the two putative lobes. To this end, we used the catalogue compiled by Flesch \& Hardcastle $(2004)^{2}$. For a typical large-scale double, NVSS carried out with the VLA in D-conf. is well suited to indicate the presence of the lobes regardless of whether they are relics or not. This way, the algorithm associating optically-selected AGNs with doublelobed radio sources used by Flesch \& Hardcastle works reliably even if a given lobe is a relic. From this point on, the selection process was based on the elementary principle of radio interferometry: diffuse objects were poorly imaged without short spacings. Specifically, we relied upon the assumption that VLA in B-conf. used during the Faint Images of the Radio Sky at Twenty-Centimeters survey (FIRST) (White et al. 1997) ${ }^{3}$ failed to reproduce lobes well or even at all if they were diffuse. Therefore, targets selected form NVSS embedded within FIRST footprint were processed with an algorithm calculating the ratio

\footnotetext{
1 Website: http://www.cv.nrao.edu/nvss.

2 Website: http://quasars.org/qorg-data.htm.

3 Website: http://sundog.stsci.edu.
} 
Table 1. Basic parameters of the targets.

\begin{tabular}{|c|c|c|c|c|c|c|c|}
\hline \multirow[t]{2}{*}{ Object name } & \multirow[t]{2}{*}{ RA } & \multirow[t]{2}{*}{ Dec } & \multirow[t]{2}{*}{$z$} & \multirow{2}{*}{$\begin{array}{c}\text { Projected } \\
\text { linear size } \\
{[\mathrm{kpc}]}\end{array}$} & \multirow{2}{*}{$\begin{array}{c}\text { Core flux }{ }^{a} \\
\text { [mJy] }\end{array}$} & \multicolumn{2}{|c|}{ Flux of the relic lobe } \\
\hline & & & & & & $\begin{array}{l}\text { Peak } \\
{[\mathrm{mJy}]}\end{array}$ & $\begin{array}{c}\text { Integrated } \\
{[\mathrm{mJy}]}\end{array}$ \\
\hline B $0847+548$ & 085039.981 & +543753.19 & $0.367^{b}$ & 567 & 13.49 & 1.36 & 33.69 \\
\hline $\mathrm{J} 115139.6+335541$ & 115139.677 & +335541.74 & $0.851^{b}$ & 898 & 6.58 & $-{ }^{c}$ & $-{ }^{c}$ \\
\hline $\mathrm{B} 1351+267$ & 135335.925 & +263147.54 & $0.307^{d}$ & 752 & 23.63 & 2.92 & 39.55 \\
\hline
\end{tabular}

Notes. ${ }^{(a)}$ Extracted from FIRST catalogue. ${ }^{(b)}$ Source: SDSS. ${ }^{(c)}$ Not itemised in FIRST catalogue. ${ }^{(d)}$ Source: Eracleous \& Halpern (2004).

of peak-to-integrated flux densities for FIRST catalogue items pertinent to each of the two NVSS lobes. When that ratio was very low for all the FIRST components within the given NVSS lobe, which meant that the lobe was featureless and so likely to be diffuse, it was regarded as a potential relic. Additionally, we found that in a number of cases the NVSS component had no FIRST equivalents and, obviously, we treated them as true positives.

Application of the above automated procedure led to a selection of a few hundreds of potential targets. This approach was by no means perfect and it produced quite a number of false positives. To sieve them out, we downloaded all the FIRST images indicated by the algorithm and inspected them visually. Thirtyeight sources with one relic and one "active" lobe remained. They were mainly normal galaxies without a trace of a radio core, but several sources in our sample appeared to have cores anyway and a few of them were identified with QSOs. For three, the flux densities of the cores at $1420 \mathrm{MHz}$ as seen in FIRST were more than $5 \mathrm{mJy}$, which made these objects suitable targets for observations with the European VLBI Network (EVN).

\section{Observational data}

\subsection{Radio domain}

The basic parameters of the radio sources selected for VLBI observations are given in Table 1 and their NVSS and FIRST maps are shown in Fig. 1. Based on the NVSS images, the radio structures of our targets are clearly double or triple with one lobe slightly weaker, but only the FIRST maps reveal the real cause of that weakness: lack of the hotspot and diffuse shapes. Quantitatively, the latter feature is well expressed by the ratio of peak-to-integrated flux density of the diffuse lobe as shown in the last two columns of Table 1.

The EVN observations were carried out on 2009 November 9/10 at $1658 \mathrm{MHz}$. The network consisted of 10 telescopes: Effelsberg, Lovell, Onsala, Medicina, Noto, Robledo, Shanghai, Toruń, Urumqi, and Westerbork. Two polarisations were recorded in eight $8-\mathrm{MHz}$ sub-bands each. Because our targets were weak, phase referencing was used. The scans on targets and phase calibrators were 10- and 2-min. long, respectively and were placed as evenly as possible on the $u-v$ plane. We assigned $3.5 \mathrm{~h}$ of observing time for B0847+548, $5 \mathrm{~h}$ for $\mathrm{J} 115139.6+335541$, and $4 \mathrm{~h}$ for B1351+267. The data reduction using AIPS was carried out in a standard way. Amplitude self-calibration was applied to the visibility data before obtaining the final images; they are shown in Figs. 2-4. The noise in these images is typically $10 \mu \mathrm{Jy} /$ beam.

Flux densities of the milliarcsecond structures were measured with AIPS TVSTAT utility. We also fitted single Gaussians to the brightest features in the source centres. Based on these figures, we calculated the brightness temperatures of those features using formula with Eq. (1) from Ulvestad et al. (2005). All the above data are given in Table 2. As discussed in Sect. 4.1, very high brightness temperatures are indicative of jet production, albeit these jets could be very weak. This agrees with the observed morphologies: neither of the three milliarcsecond structures are of core-jet type as in the case of the lobe-dominated RLQs - see discussion in Sect. 4.1.

\subsection{Optical domain}

Spectra of two quasars, B0847+548 and J115139.6+335541, are available in the Sloan Digital Sky Survey (SDSS) ${ }^{4}$. Both are very typical for QSOs: the continua rise towards shorter wavelengths and they are featured by both broad and narrow emission lines. For $\mathrm{B} 0847+548$, the $\mathrm{H} \beta$, and for $\mathrm{J} 115139.6+335541$ the $\mathrm{Mg}$ II line can be best used for a measurement of $\mathrm{BH}$ mass, see e.g. Czerny \& Nikołajuk (2010) for an up-to-date review. The BH masses and related quantities for 105,783 quasars in the SDSS Data Release 7 (Abazajian et al. 2009) quasar catalogue (Schneider et al. 2010) have been recently calculated by Shen et al. (2010). We extracted the BH masses, bolometric luminosities, and Eddington ratios for B0847+548 and $\mathrm{J} 115139.6+335541$ from that catalogue and listed them in Table 3. At the time of writing, the results of Shen et al. (2010) had not been accepted for publication yet so independent calculations were carried out by Nikołajuk (private communication). His results were very close to those of Shen et al. (2010). Unfortunately, no B1351+267 spectrum was available in the literature (Eracleous \& Halpern 2004, quoted only the redshift for this object). Therefore, we were unable to give the $\mathrm{BH}$ mass and Eddington ratio for it.

\section{Discussion}

\subsection{Cores of RLQs vs. cores of RQQs}

Radio structures of QSOs are highly influenced by orientation effects as predicted by the AGN unification scheme. As a result, depending on the angle between the line of sight and the jets, RLQs can be perceived either as core- (beamed) or lobe-dominated (unbeamed). Doppler beaming and boosting makes the first group ideal objects for VLBI studies like, e.g. the Caltech-Jodrell Bank Flat-spectrum (CJF) sample survey (Taylor et al. 1996) that encompasses 293 sources. Following the predictions based on the unification scheme, the core-jet structure overwhelmingly dominates them. On the other hand, cores of 25 lobe-dominated QSOs from 3CR-based complete sample were comprehensively investigated by Hough et al. (2002), who observed them in one or more epochs between 1981 and 1997 with various VLBI arrays at several frequencies between 5

4 Website: http://www.sdss.org/ 

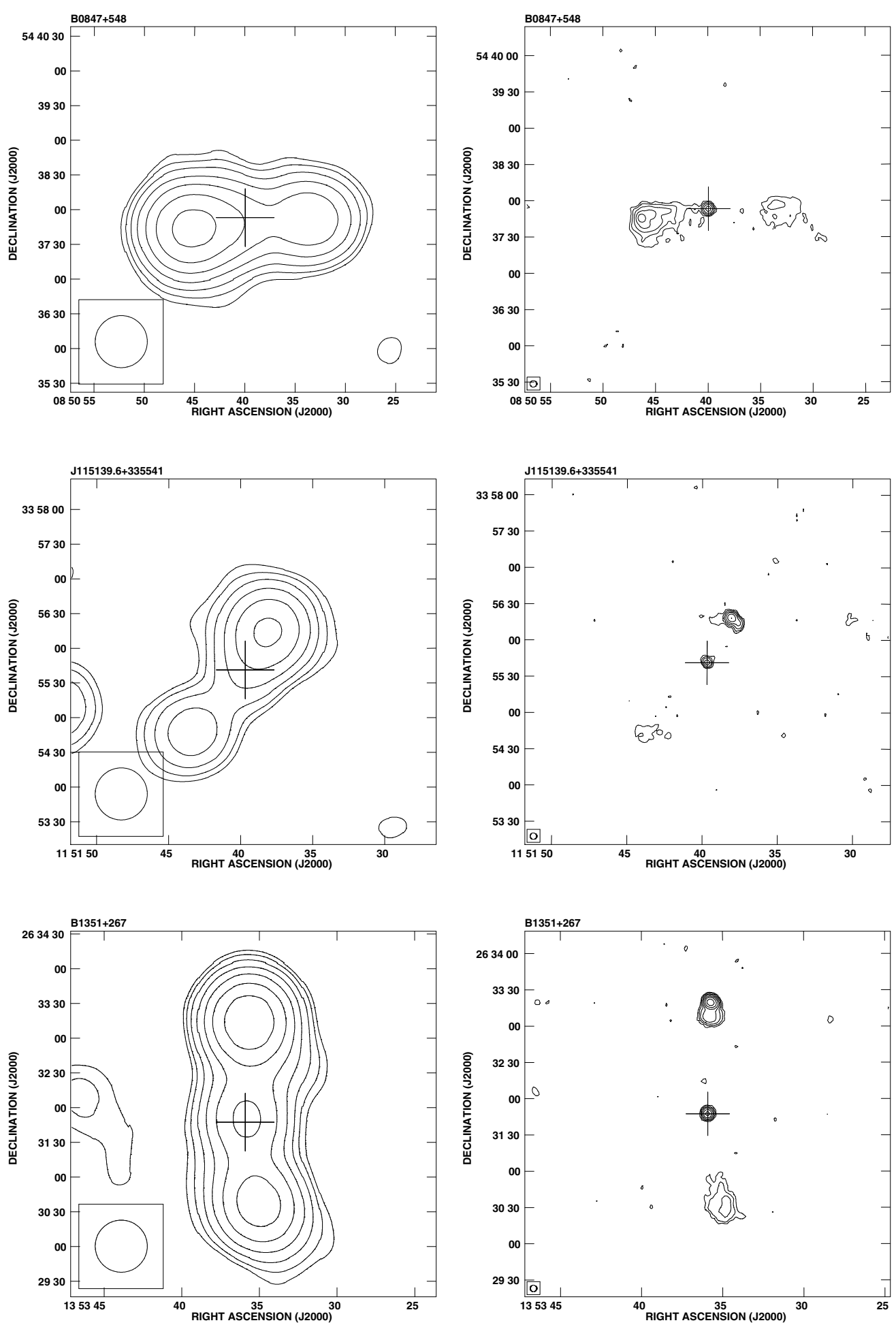

Fig. 1. NVSS (left column) and FIRST (right column) images of three quasars with asymmetric lobes. Contours are increased by a factor of 2; the first contour level corresponds to $1 \mathrm{mJy} /$ beam for NVSS images and $0.5 \mathrm{mJy} / \mathrm{beam}$ for FIRST images. Crosses indicate the positions of optical objects.

and $22 \mathrm{GHz}$. Later, these observations were supplemented with VLBA and High Sensitivity Array (HSA) observations (Hough 2008) so eventually the nuclei in all 25 objects were imaged. Cores in three of them were unresolved and the remaining 22 showed one-sided jets located on the same side of the compact core as the one-sided large-scale jets seen in the VLA images.
Given that no counter-jets were observed, the authors estimated that these objects were all orientated within $\sim 70^{\circ}$ to the line of sight. Their results were consistent with orientation-dependent relativistic beaming effects and unification of core- and lobedominated QSOs. In the latter class, the presence of jets is a clear signature that the objects belonging to it are not only apparently 


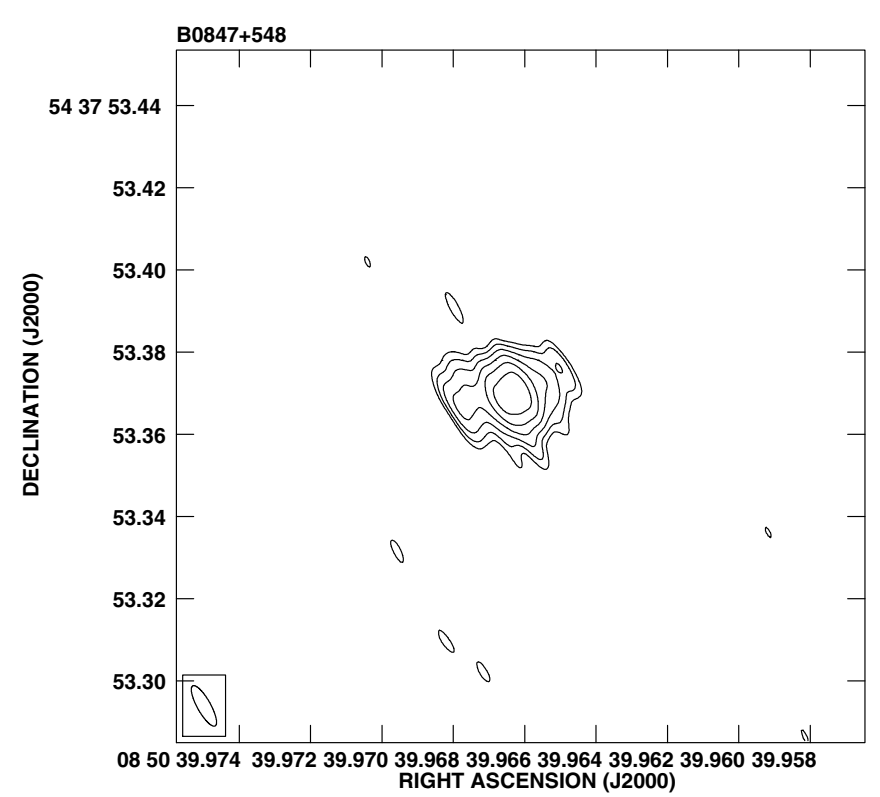

Fig. 2. EVN image of the core of B0847+548. Contours are increased by a factor of 2 ; the first contour level corresponds to $5 \sigma$ level which is $52 \mu \mathrm{Jy} / \mathrm{beam}$. The peak flux density is $2.84 \mathrm{mJy} / \mathrm{beam}$. The beam size is $11.2 \times 3.2$ milliarcsec at the position angle of $29^{\circ}$.

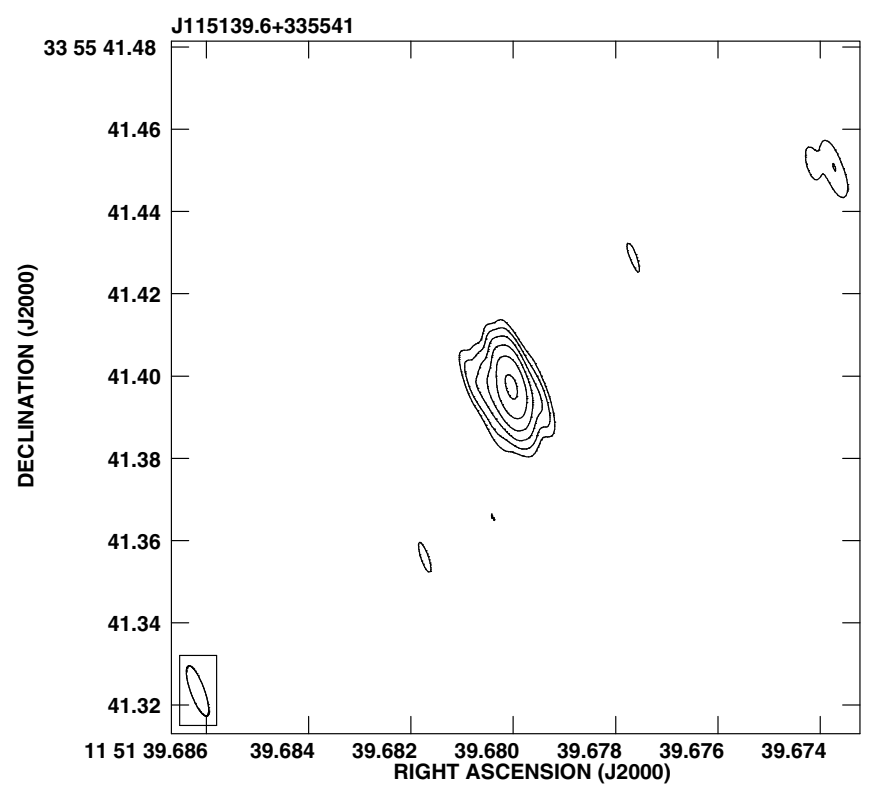

Fig. 3. EVN image of the core of J115139.6+335541. Contours are increased by a factor of 2 ; the first contour level corresponds to $5 \sigma$ level which is $51 \mu \mathrm{Jy} / \mathrm{beam}$. The peak flux density is $1.85 \mathrm{mJy} / \mathrm{beam}$. The beam size is $12.9 \times 3.4$ milliarcsec at the position angle of $20^{\circ}$.

RL because of the lobes' dominance, but are truly RL, i.e. the energy transport from the core to the lobes, which is the prime cause of the existence of radio structures, is ongoing.

By definition, RQQs are devoid of radio lobes because their nuclei either do not produce jets, or the jets they produce are too weak to inflate lobes. This does not preclude, however, the existence of weak radio cores there. Indeed, they are observed

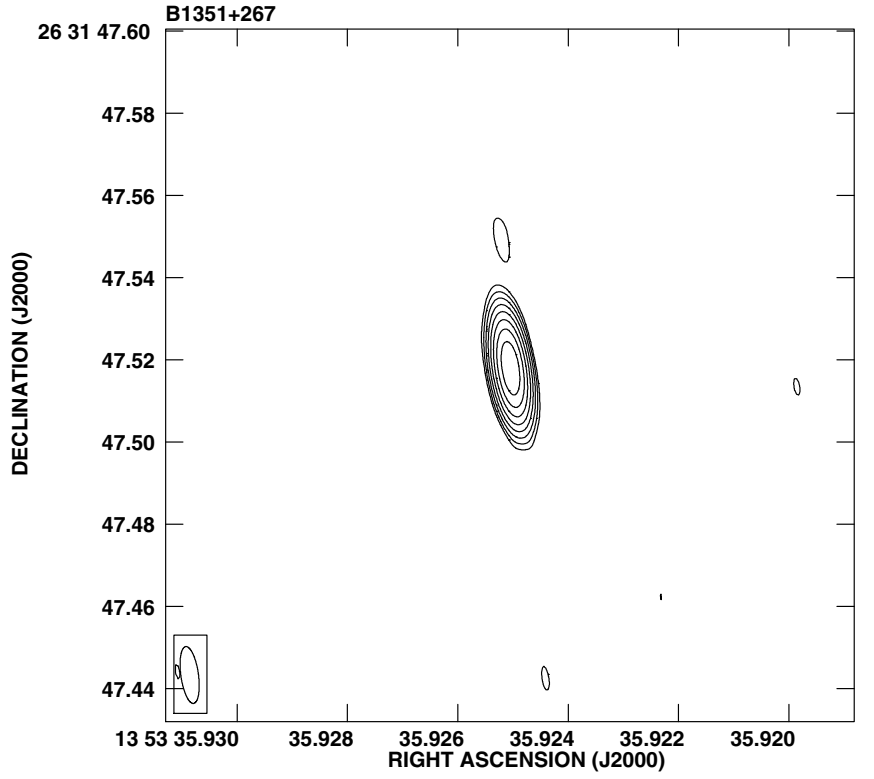

Fig. 4. EVN image of the core of B1351+267. Contours are increased by a factor of 2 ; the first contour level corresponds to $5 \sigma$ level which is $56 \mu \mathrm{Jy} /$ beam. The peak flux density is $13.1 \mathrm{mJy} /$ beam. The beam size is $13.9 \times 4.3$ milliarcsec at the position angle of $8^{\circ}$.

and the question that immediately emerges is whether that radio emission is associated with starbursts (see e.g. Sopp \& Alexander 1991) or with weak-jet producing central engines. Blundell \& Beasley (1998) sought the answer for this question by means of the VLBA observations of 12 RQQs, because the detection of milliarcsecond structure implies that the brightness temperatures of the emission is $T_{\mathrm{B}} \gtrsim 10^{6} \mathrm{~K}$, while for typical supernova remnants $T_{\mathrm{B}} \lesssim 10^{5} \mathrm{~K}$. Although the structures that they managed to map out were only indicative of jets, the measurements of brightness temperatures provided strong evidence for jet-producing central engines in eight sources. Further investigations of RQQ cores by means of the VLBA observations were carried out by Ulvestad et al. (2005). They observed six RQQ cores and got images for five of them: four were dominated by unresolved component, while the fifth showed quite a rich structure that was labelled "two-sided jet" by the authors. Note that the structure had a steep spectrum. Being aware of that and expecting that our sources can also bear this feature, we decided to make our observations at $1.6 \mathrm{GHz}$ to attain better signal-to-noise ratios at the cost of lower resolution.

The brightness temperatures measured by Ulvestad et al. (2005) were of the order of $10^{8}$ or greater, ruling out the thermal origin of the observed radiation. Thus, the authors confirmed the hypothesis of Miller et al. (1993) that radio cores of RQQs were fundamentally similar to those in RLQs, while the RQQ jets were simply less powerful. The more general conclusion that emerges from their work - but also from Blundell et al. (1996); Blundell \& Beasley (1998); Blundell et al. (2003) - is that the term "RQQ" should not be understood in a verbatim manner, namely an RQQ is not necessarily a QSO that is completely "silent", but weak in radio domain (Barvainis et al. 2005). What is much more important instead is that central engines of RQQs do not produce jets strong enough to transport the energy to the lobes. This agrees with the hypothesis put forward by Ghisellini et al. (2004) that all black hole/accretion disc systems in AGNs 
Table 2. Observed parameters of the milliarcsecond structures.

\begin{tabular}{lllc}
\hline \hline Object name & \multicolumn{2}{c}{ Flux } & density \\
& $\begin{array}{c}\text { otal }^{a} \\
{[\mathrm{mJy}]}\end{array}$ & $\begin{array}{c}\text { Fitted }^{b} \\
{[\mathrm{mJy}]}\end{array}$ & $\begin{array}{c}\text { Brightness } \\
\text { temperature }^{c} \\
{[\mathrm{~K}]}\end{array}$ \\
\hline B0847+548 & 10.0 & 8.3 & $6.65 \times 10^{8}$ \\
J115139.6+335541 & 4.2 & 3.8 & $1.55 \times 10^{8}$ \\
B1351+267 & 13.7 & 13.7 & $7.73 \times 10^{9}$ \\
\hline
\end{tabular}

Notes. ${ }^{(a)}$ Measured with AIPS TVSTAT utility. ${ }^{(b)}$ Fitted to the strongest feature. ${ }^{(c)}$ Based on the flux density of the strongest feature.

Table 3. BH masses, bolometric luminosities, and Eddington ratios.

\begin{tabular}{lccc}
\hline \hline Object name & $\log \left(M_{\mathrm{BH}} / M_{\odot}\right)$ & $\begin{array}{c}\log \left(L_{\mathrm{bol}}\right) \\
{[\log (\mathrm{erg} / \mathrm{s})]}\end{array}$ & $L_{\mathrm{bol}} / L_{\mathrm{Edd}}$ \\
\hline $\mathrm{B} 0847+548$ & 9.406 & 45.541 & 0.011 \\
$\mathrm{~J} 115139.6+335541$ & 9.383 & 46.272 & 0.061 \\
\hline
\end{tabular}

can produce some kind of outflow or jet, but only in a minority of cases, i.e. in truly radio-loud objects, the jet is successfully launched and accelerated to relativistic speeds. In the majority of cases, the jet is "aborted", yet it is responsible for a relatively weak radio emission.

Our findings are fully consistent with those by Ulvestad et al. (2005) both in terms of brightness temperatures and milliarcsecond-scale morphologies: B1351+267 is point-like, $\mathrm{J} 115139.6+335541$ is nearly point-like with two protrusions on the eastern and western side, and B0847+548 has two-sided mini-jets. All three have low radio flux densities and yet high brightness temperatures. Altogether, the galactic nuclei we imaged with the EVN are characteristic of RQQs. The major difference, though, is pertinent to the large-scale overall radio structures: while the objects observed by Ulvestad et al. (2005) are truly RQ, ours are classified as RL because of the presence of the lobes. Nevertheless, we claim that the cores of the three quasars we deal with here not only resemble those in RQQs, but belong to the RQQ category and so these quasars are observed after a recent transition between the RL and RQ states. Their cores herald the onset of the RQ state, whereas the lobes, while still rendering the RL label formally applicable to these quasars, are actually only remnants of the former RL state. On the other hand, we find that our quasars do not fit to the model by Blundell \& Kuncic (2007) because it requires mass accretion rates substantially higher than Eddington, which is not the case, see Table 3. (This model has also been criticised on theoretical grounds by Laor \& Behar 2008.)

\subsection{Quasar-microquasar analogy}

Although few XRBs are persistent X-ray sources, the majority are variable. The analysis of their light curves at different X-ray bands shows a clear correlation between the luminosity and hardness, which led to distinction of two basic states in XRBs: high/soft (HS) and low/hard (LH). The $1-10 \mathrm{keV}$ radiation observed in the HS state is best explained as a thermal emission form the accretion disc, while the $20-100 \mathrm{keV}$ radiation of the LH state, when the spectrum becomes non-thermal with a typical photon index $\Gamma \sim 1.7$ and an exponential cutoff around $100 \mathrm{keV}$, is a result of Comptonisation of softer photons in the accretion disc corona of high-energy electrons. More precisely, the LH state can be regarded as a follow-up of the HS state switch-off: when the density of soft seed photons is greatly reduced, the resultant Comptonised spectrum hardens, see e.g. McClintock \& Remillard (2004) for a review. Three additional states: "quiescent", "very high" (VH), and "intermediate" have been all identified for GX 339-4 by Markert et al. (1973), Miyamoto et al. (1991), and Méndez \& van der Klis (1997), respectively. At least some of these three can be observed in other XRBs. Esin et al. (1997) attributed these five states - in order of increasing luminosity these were: the quiescent, LH, intermediate, $\mathrm{HS}$, and $\mathrm{VH}$ - to different accretion rates, although the VH state could not be unified within the framework of their model of accretion flows around BHs. Also, according to them, different accretion modes were at work for particular states: in quiescent, LH, and intermediate states, the radiatively inefficient advection-dominated accretion flow (ADAF) was present, while for $\mathrm{HS}$ and $\mathrm{VH}$ the ADAF (inner) zone disappeared and the radiatively efficient accretion disc extended down to the innermost stable circular orbit (ISCO) around the $\mathrm{BH}$.

The correlation between the X-ray-emission-based states in $\mathrm{XRBs}$ and their radio properties is particularly strong: the radio emission is generated in LH and VH states, while it drops in the HS state below detectable levels, most likely because of the physical disappearance of jets, see Fender (1999) for a brief summary. The parallel existence of a clear RL/RQ dichotomy in XRBs and AGNs immediately leads to attempts to unify XRBs and AGNs based on their radio properties, i.e. to identify specific states observed in XRBs with classes of AGNs (Meier 2001; Maccarone et al. 2003; Falcke et al. 2004). The following picture emerges: Sgr A*, LINERs, FR I radio galaxies, and BL Lac objects remain in a state analogous to the LH state of XRBs, RQQs and Seyfert galaxies represent the HS state, whereas FR II radio galaxies and RLQs behave as if they were in the VH state. More recently, this scheme was advanced by Wu \& Cao (2008) who showed that FR I and FR II radio galaxies could have different accretion modes and the accretion mode in the majority of FR I galaxies could be of ADAF type similar to that in the LH state in XRBs. On the other hand, many RL objects had both the most powerful known radio jets and optical properties typical for HS state so the VH state, which is the only state where these two features could be combined, should be attributed to them.

In the framework of the above paradigm, the objects we observed, which are effectively RQQs based on the status of their nuclei, are in a state analogous to the HS state but given the presence of decaying large-scale radio structures, they must have entered that state only recently. This immediately leads to a conjecture that migration of an AGN to the analog of the HS state may be a part of a more general evolutionary process. Taking into account all the similarities between XRBs and AGNs, such an evolution - which is sped up by the factor of the AGN-to-XRB $\mathrm{BH}$ mass ratio - should possibly be followed directly in XRBs.

\subsection{XRBs and AGNs - common evolution?}

A new light on the XRB/AGN unification was shed by Fender et al. (2004), but see also a simplified version of that paper: Fender et al. (2005), who constructed a semi-quantitative model of XRB evolution. Piecing together XRB observational data and placing them on the hardness-intensity diagram (HID), they found an evolutionary path of XRBs, sometimes called the "turtle head" diagram - see Fig. 7 in Fender et al. (2004). The XRB timeline starts at the quiescence stage, then moves nearly vertically during the LH state. When it leaves that state, it turns left towards the $\mathrm{VH}$ state. At some point, usually corresponding to the peak of the VH state, the photon index $\Gamma$ rapidly increases, producing an internal shock in the outflow and the jet properties 
change, most notably its velocity. The final, most powerful jet has the highest Lorentz factor, causing the propagation of an internal shock through the slower-moving outflow in front of it. Following that, an XRB enters the HS stage when no jet is produced although some XRBs, like GRS 1915+105, make repeated excursions back to VH state (see e.g. Sikora et al. 2007, and references therein). After a number of "loops" in the HID (if any), an XRB enters the HS state permanently and returns, via intermediate state, back to quiescence.

The above path can be followed directly for XRBs because they evolve quickly enough, but given the identity of both the accretion process (McHardy et al. 2006) and the jet generation mechanism in microquasars and quasars (Türler et al. 2004) one should expect that this path is common to AGNs, albeit it is impossible to be followed within a human lifetime because of the timescaling factor, which reaches several orders of magnitude. What could be done, however, is to produce a HID ana$\log$ for AGNs and check on a statistical basis if it resembles the HID for XRBs. This task was accomplished by Körding et al. (2006). Because the blackbody temperature of the accretion disc scales with black hole mass as $M^{-1 / 4}$, the SEDs of accretion discs around SMBHs peak in the optical/UV domain, not the $\mathrm{X}$-ray, and a HID for AGNs would not contain any information about the disc emission. Therefore, instead of a HID, the authors constructed a disc-fraction/luminosity diagram (DFLD) plotting $L_{\mathrm{D}}+L_{\mathrm{PL}}$ against $L_{\mathrm{PL}} /\left(L_{\mathrm{D}}+L_{\mathrm{PL}}\right)-$ where $L_{D}$ denoted the disc luminosity and $L_{\mathrm{PL}}$ the luminosity of the power-law component - for a sample of 4963 SDSS Data Release 5 quasars. What they found was a striking similarity between the DFLD for AGNs and DFLD for a simulated sample of XRBs. This shows that in general a large population of AGNs follow the same pattern as XRBs. Consequently, not only particular classes of AGNs could be assigned to different XRB states in a "static" manner (Meier 2001; Maccarone et al. 2003; Falcke et al. 2004), but also signatures of transitions between those states could be looked for in AGNs. In particular, thanks to the long decay times of the radio lobes (Komissarov \& Gubanov 1994), a recent transition from RL VH state to RQ HS state should be attributed to the objects with already radio-quiet cores and still observable relic radio lobes. The existence of these objects has been predicted by Fender et al. (2005). We claim that the quasars with asymmetric overall radio structures we presented here fit those predictions.

Finally, it is worth mentioning at this point that our attempt to identify a transition in accretion properties of AGNs with a respective transition between spectral states of XRBs is not the first endeavour of this kind. Marchesini et al. (2004) suggested that the transition of accretion properties among different types of RL AGNs alone could also have its analog in the XRB domain.

\section{Summary and final conclusion}

The unification of XRBs and AGNs has recently gained a substantial boost. A major step towards a full unification between these two classes were made by Fender et al. (2004), who showed an evolutionary sequence for XRBs in the HID and by Körding et al. (2006) who constructed a DFLD - an analog of HID applicable for AGNs. One cannot directly show that AGNs are moving along an evolutionary track on a DFLD but, given that many characteristics of XRBs and AGNs have been found to be either analogous or identical, is it very reasonable to expect that the evolutionary sequence traced for XRBs should also be valid for AGNs. In this work, we showed the presence of transitions from the RL to the RQ state in three quasars, using their large-scale radio structures as a historical record of the former RL state and the micro-scale radio structures as an evidence of the present RQ state. If, in line with Nipoti et al. (2005), the $\mathrm{XRB} / \mathrm{AGN}$ analogy indeed holds also for the evolution of these two classes of object, the quasars we investigated could be regarded as objects after a recent transition from VH to HS state.

The RL-to-RQ transition scenario outlined here has yet another consequence. According to the unification scheme (Antonucci 1993; Urry \& Padovani 1995), the RL/RQ dichotomy should be attributed to the presence or absence of $\mathrm{BH}$ spin, respectively. While small $\mathrm{BH}$ spins can still be the reason why some AGNs are RQ, according to some studies (Sikora et al. 2007, and references therein), BHs in both RLQs and RQQs may have large spins. For example, according to Elvis et al. (2002) most SMBHs rotate rapidly. The same conclusion has been reached by Wang et al. (2006) based on an investigation of 12698 quasars from SDSS. The BHs in the three quasars we deal with here must have large spins to make it possible for these objects to have been RL in the past. They have become RQ now, but it is hard to find a mechanism forcing their BHs to spin down during such a transition. Eventually, when their lobes fade out completely, they will turn out to be bona fide RQQs, yet their BHs will still have large spins contrary to the paradigm.

Acknowledgements. A.M. is very grateful to Marek Sikora and also to Bożena Czerny for inspiring discussions and particularly to Marek Nikołajuk for his help with the interpretation of the optical spectra and independent calculation of SMBH masses in the objects we investigated.

The European VLBI Network is a joint facility of European, Chinese, South African and other radio astronomy institutes funded by their national research councils.

This research has made use of the NASA/IPAC Extragalactic Database (NED), which is operated by the Jet Propulsion Laboratory, California Institute of Technology, under contract with the National Aeronautics and Space Administration.

Funding for the SDSS and SDSS-II has been provided by the Alfred P. Sloan Foundation, the Participating Institutions, the National Science Foundation, the US Department of Energy, the National Aeronautics and Space Administration, the Japanese Monbukagakusho, the Max Planck Society, and the Higher Education Funding Council for England. The SDSS Web Site is http://www . sdss.org/. The SDSS is managed by the Astrophysical Research Consortium for the Participating Institutions. The Participating Institutions are the American Museum of Natural History, Astrophysical Institute Potsdam, University of Basel, University of Cambridge, Case Western Reserve University, University of Chicago, Drexel University, Fermilab, the Institute for Advanced Study, the Japan Participation Group, Johns Hopkins University, the Joint Institute for Nuclear Astrophysics, the Kavli Institute for Particle Astrophysics and Cosmology, the Korean Scientist Group, the Chinese Academy of Sciences (LAMOST), Los Alamos National Laboratory, the Max-Planck-Institute for Astronomy (MPIA), the Max-Planck-Institute for Astrophysics (MPA), New Mexico State University, Ohio State University, University of Pittsburgh, University of Portsmouth, Princeton University, the United States Naval Observatory, and the University of Washington.

\section{References}

Abazajian, K. N., Adelman-McCarthy, J. K., Agüeros, M. A., et al. 2009, ApJS, 182,543

Alexander, P., \& Leahy, J. P. 1987, MNRAS, 225, 1

Antonucci, R. 1993, ARA\&A, 31, 473

Barthel, P. D. 1989, ApJ, 336, 606

Barvainis, R., Lehár, J., Birkinshaw, M., Falcke, H., \& Blundell, K. M. 2005, ApJ, 618, 108

Blundell, K. M., \& Beasley, A. J. 1998, MNRAS, 299, 165

Blundell, K. M., \& Kuncic, Z. 2007, ApJ, 668, L103

Blundell, K. M., Beasley, A. J., Lacy, M., \& Garrington, S. T. 1996, ApJ, 468, L91

Blundell, K. M., Beasley, A. J., \& Bicknell, G. V. 2003, ApJ, 591, L103

Castro-Tirado, A. J., Brandt, S., \& Lund, N. 1992, IAU Circ., 5590, 2

Chatterjee, R., Marscher, A. P., Jorstad, S. G., et al. 2009, ApJ, 704, 1689

Condon, J. J., Cotton, W. D., Greisen, E. W., et al. 1998, AJ, 115, 1693

Czerny, B., \& Nikołajuk, M. 2010, Mem. Soc. Astron. Ital., 81, 281 
Elvis, M., Risaliti, G., \& Zamorani, G. 2002, ApJ, 565, L75 Eracleous, M., \& Halpern, J. P. 2004, ApJS, 150, 181

Esin, A. A., McClintock, J. E., \& Narayan, R. 1997, ApJ, 489, 865

Falcke, H., Körding, E. G., \& Markoff, S. 2004, A\&A, 414, 895

Fanaroff, B. L., \& Riley, J. M. 1974, MNRAS, 167, 31P

Fender, R. P. 1999, ESO workshop Black Holes in binaries and galactic nuclei, ed. L. Kaper, E. P. J. van den Heuvel, \& P. A. Woudt (Springer-Verlag) [arXiv: astro-ph/9911176]

Fender, R., \& Belloni, T. M. 2004, ARA\&A, 42, 317

Fender, R. P., Belloni, T. M., \& Gallo, E. 2004, MNRAS, 355, 1105

Fender, R. P., Belloni, T. M., \& Gallo, E. 2005, Ap\&SS, 300, 1

Fender, R. P., Körding, E. G., Belloni, T., et al. 2007, VI Microquasar Workshop, ed. T. Belloni, PoS(MQW6)011 [arXiv: 0706. 3838]

Flesch, E., \& Hardcastle, M. J. 2004, A\&A, 427, 387

Ghisellini, G., Haardt, F., \& Matt, G. 2004, A\&A, 413, 535

Hough, D. 2008, Extragalactic Jets: Theory and Observation from Radio to Gamma Ray, ed. T. A. Rector, \& D. S. De Young, ASPC, 386, 274

Hough, D. H., Vermeulen, R. C., Readhead, A. C. S., et al. 2002, AJ, 123, 1258

Kaiser, C. R., Schoenmakers, A. P., \& Röttgering, H. J. A. 2000, MNRAS, 315, 381

Komissarov, S. S., \& Gubanov, A. G. 1994, A\&A, 285, 27

Körding, E. G., Jester, S., \& Fender, R. P. 2006, MNRAS, 372, 1366

Laor, A., \& Behar, E. 2008, MNRAS, 390, 847

Liu, R., Pooley, G., \& Riley, J. M. 1992, MNRAS, 257, 545

Maccarone, T. J., Gallo, E., \& Fender, R. 2003, MNRAS, 345, L19

Machalski, J., Kozieł-Wierzbowska, D., Jamrozy, M., \& Saikia, D. J. 2008, ApJ, 679,149

Marecki, A., Kunert-Bajraszewska, M., \& Spencer, R. E. 2006, A\&A, 449, 985

Marchesini, D., Celotti, A., \& Ferrarese, L. 2004, MNRAS, 351, 733
Markert, T. H., Canizares, C. R., Clark, G. W., et al. 1973, ApJ, 184, L67 Marscher, A. P., Jorstad, S. G., Gómez, J.-L., et al. 2002, Nature, 417, 625 McClintock, J. E., \& Remillard, R. A. 2004, in Compact Stellar X-ray Sources, ed. W. H. G. Lewin, \& M. van der Klis (Cambridge University Press) [arXiv: astro-ph/0306213]

McHardy, I. M., Körding, E. G., Knigge, C., Uttley, P., \& Fender, R. P. 2006, Nature, 444, 730

Meier, D. L. 2001, ApJ, 548, L9

Méndez, M., \& van der Klis, M. 1997, ApJ, 479, 926

Miller, P., Rawlings, S., \& Saunders, R. 1993, MNRAS, 263, 425

Mirabel, I. F., \& Rodríguez, L. F. 1994, Nature, 371, 46

Mirabel, I. F., Rodríguez, L. F., Cordier, B., Paul, J., \& Lebrun, F. 1992, Nature, 358,215

Mirabel, I. F., Rodríguez, L. F., Marti, J., et al. 1993, IAU Circ., 5773, 2

Miyamoto, S., Kimura, K., Kitamoto, S., Dotani, T., \& Ebisawa, K. 1991, ApJ, 383,784

Nipoti, C., Blundell, K. M., \& Binney, J. 2005, MNRAS, 361, 633

Shakura, N. I., \& Sunyaev, R. A. 1976, MNRAS, 175, 613

Shen, Y., Hall, P. B., Richards, G. T., et al. 2010 [arXiv: 1006. 5178]

Schneider, D. P., Richards, G. T., Hall, P. B., et al. 2010, AJ, 139, 2360

Sikora, M., Stawarz, Ł., \& Lasota, J.-P. 2007, ApJ, 658, 815

Sopp, H. M., \& Alexander, P. 1991, MNRAS, 251, 14P

Taylor, G. B., Vermeulen, R. C., Readhead, A. C. S., et al. 1996, ApJS, 107, 37

Türler, M., Courvoisier, T. J.-L., Chaty, S., \& Fuchs, Y. 2004, A\&A, 415, L35

Ulvestad, J. S., Antonucci, R. R. J., \& Barvainis, R. 2005, ApJ, 621, 123

Urry, C. M., \& Padovani, P. 1995, PASP, 107, 803

Wang, J.-M., Chen, Y.-M., Ho, L. C., \& McLure, R. J. 2006, ApJ, 642, L111

White, R. L., Becker, R. H., Helfand, D. J., \& Gregg, M. D. 1997, ApJ, 475, 479

Wu, Q., \& Cao, X. 2008, ApJ, 687, 156 